\title{
Phospho-proteomic analysis of the effect of merecidin inhibiting the human lung adenocarcinoma A549 cells
}

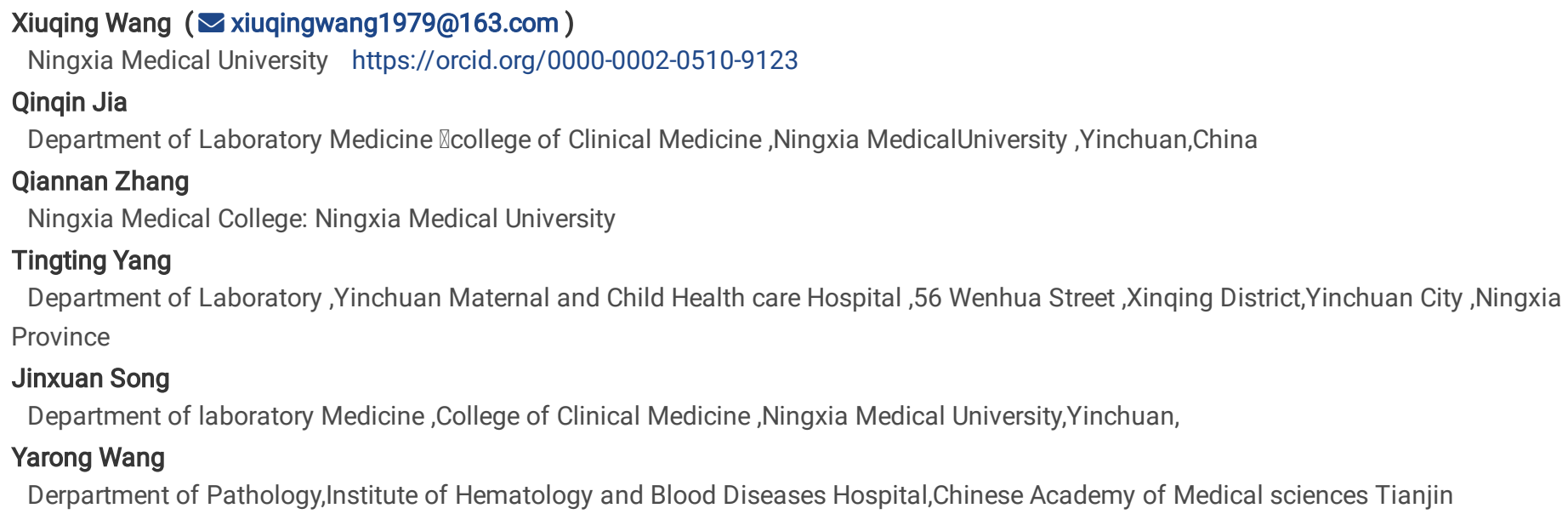

\section{Research}

Keywords: phospho-proteomics, antibacterial peptide, merecidin, lung adenocarcinoma

Posted Date: April 21st, 2021

DOI: https://doi.org/10.21203/rs.3.rs-378437/v2

License: (c) (7) This work is licensed under a Creative Commons Attribution 4.0 International License. Read Full License 


\section{Abstract}

Background: Merecidin induced A549 cells apoptosis. The present study aimed to assess the differentially phosphorylated proteins in lung cancer A549 cells treated with antibacterial peptide merecidin.

Methods: TMT/iTRAQ labeling, LC-MS/MS analysis, HPLC grading, IMAC modification and enrichment, and liquid chromatography-mass spectrometry of peptide. The data of proteins were filtered according to the localization probability $>0.75$ standard. The phosphorylated proteomics data were analyzed using GO, KEGG, and STRING databases. Western blots examined the changes in phosphorylated protein expression.

Results: Protein function enrichment showed significant changes in the phosphorylation level with respect to protein binding, metabolic activity, molecular function regulation, cell process, and biological function regulation. Integrated pathway bioinformatics results showed that differential proteins are associated with several pathways, including mTOR and AMPK etc. Screening of the COG database revealed differentiated phosphorylated proteins in cell signal transduction, RNA transcription, translation processing and modification, ribosome synthesis of proteins, cytoskeleton protein formation, intracellular material transport, secretion, and vesicle transport. Protein interaction level analysis identified an interaction network with HDAC1, RPL23A, SRSF3H, and NCBP1.etc. The phosphorylated proteomics data and showed that after merecidin treatment, ATG2B and ULK1s were significantly upregulated, while MAPK1 and AKT were significantly downregulated. In addition, Western blot also showed the upregulated level of ATG13,ULK1;MAPK1 downregulated.

Conclusion: The results verified the feasibility of phosphorylated proteomics analysis, confirmed the signaling pathways, and suggested that merecidin may induce autophagy of lung adenocarcinoma A549 cells.

\section{Background}

Lung cancer has a very high incidence and rate of mortality ${ }^{1}$ worldwide, accounting for more than $16.7 \%$ of the cancer-related deaths ${ }^{2}$. Small-cell lung cancer (SCLC) represents $13-20 \%$ of lung cancer ${ }^{3-5}$. However, since 1997, there has not been any improvement in the response rates to computed tomography (CT) or survival of SCLC patients ${ }^{6,7}$. The 5 -year survival rate is less than $20 \%{ }^{7}$. Therefore, anti-cancer drugs targeting lung cancer are urgently required. Protein phosphorylation plays a significant role in many cell processes, such as proliferation, differentiation, migration, autophagy, and apoptosis.

Protein phosphorylation is one of the most important covalent modifications in organisms. The simple, flexible, and reversible properties of phosphorylation, as well as the availability of phosphate donor ATP, make phosphorylation the most common regulatory mechanism in eukaryotic cells ${ }^{8}$. Protein phosphorylation ${ }^{9}$ is a reversible process, almost regulates cell proliferation, development, differentiation, and the regulation of the cytoskeleton, apoptosis, autophagy ${ }^{10,11}$, and neural activity. An increasing number of studies have shown that proteomics analysis $^{12}$ of tumor samples is a comprehensive and dynamic method. The combination of these methods with molecular networks in cancer development elucidates the pathogenesis of tumors with respect to diagnosis, treatment, and prognosis of the disease. Several studies ${ }^{13-15}$ have analyzed the phosphorylated proteomics data of cancer cells.

LL-37 is the only antimicrobial peptide found in the human body, which is expressed in testis, skin ${ }^{16}$, nasal mucosa, saliva ${ }^{17}$, and gastrointestinal tract $^{18}$. It is a multifunctional peptide ${ }^{19}$ with antibacterial and anti-cancer properties and is also involved in the regulation of adaptive immunity 20,21 . LL-37 alpha-helix structure combined with cell membrane lipid induces the formation of cell membrane channel 22 or increases the permeability of cell membrane ${ }^{23,24}$, leading to cell death ${ }^{25,26}$. Ren et al. found that the treatment of colon cancer cells with LL-37 exposes it to anionic phosphatidylserine (PS) and DNA fragmentation, suggesting apoptosis ${ }^{27}$. Furthermore, it plays an anti-cancer role in gastric cancer ${ }^{28}$, acute myeloid leukemia ${ }^{29}$, and lymphocytic leukemia ${ }^{30}$, and also upregulates the mRNA expression and protein level of VEGF-A and the phosphorylated levels of ERK1/2 and NF-kB p65 in HPL cells ${ }^{31}$. Merecidin (also known as 17BIPHE2) is a cutting peptide of antibacterial peptide LL-37. It exerts lower hemolytic activity and less toxicity to the human body, and is superior to its parent LL-37 ${ }^{32}$ in terms of immune regulation and inhibition of various bacteria.Our previous study demonstrated that merecidin promotes lung adenocarcinoma A549 cell apoptosis ${ }^{33-35}$ and inhibits the formation of Staphylococcus aureus and Pseudomonas aeruginosa biofilm ${ }^{36,37}$; however, the underlying molecular mechanism is not yet fully understood.

\section{Methods}

\subsection{Cell culture}

The human lung adenocarcinoma cancer cell line A549 was obtained from the Affiliated Hospital of the Ningxia Medical University and cultured in in Roswell Park Memorial Institute (RPMI)-1640 medium (HyClone) containing 10\% fetal bovine serum (FBS, Gibco, USA) and 100 U/mL 
penicillin and streptomycin (Gibco) at $37^{\circ} \mathrm{C}$ in a cell culture incubator with $5 \% \mathrm{CO}_{2}$.

\subsection{Synthesis and characterization of merecidin}

The LL-37 peptide contains 37 amino acids, with two leucine residues at the N-terminus. The minimum sequence responsible for the antimicrobial effect of LL-37 was deduced from peptide activity assays and was found to be the 17-29 or 18-29 amino acids (amino acid sequence: GBKRLVQRLKDBLRNLV, L is D-type amino acid, B is biphenyl phenylalanine, and the molecular weight is $2561.866^{38,39}$ ). Solid-phase chemistry was used to synthesize the peptides with $\geq 95 \%$ purity. $\mathrm{ddH}_{2} \mathrm{O}$ solution was used in all the experiments.

\subsection{Protein extraction}

After treatment with $9 \mathrm{mmol} / \mathrm{L}$ merecidin for $6 \mathrm{~h}, \mathrm{~A} 549$ cells were sonicated three times on ice using a high-intensity ultrasonic processor (Scientz,Shanghai,China) in lysis buffer (8 M urea, 1\% protease inhibitor cocktail). For PTM (Protein Post-translational Modification) experiments, inhibitors were added to the lysis buffer ( $3 \mu \mathrm{M}$ TSA and $50 \mathrm{mM}$ NAM for acetylation). The remaining debris was removed by centrifugation at $12,000 \mathrm{~g}$ at $4^{\circ} \mathrm{C}$ for $10 \mathrm{~min}$. Finally, the supernatant was collected, and the protein concentration was determined using the BCA assay (Beyotime, Shanghai, China), according to the manufacturer's instructions.

\subsection{Trypsin digestion of the proteins}

For digestion, the protein solution was reduced with $5 \mathrm{mM}$ dithiothreitol for $30 \mathrm{~min}$ at $56^{\circ} \mathrm{C}$ and alkylated with $11 \mathrm{mM}$ iodoacetamide for $15 \mathrm{~min}$ at room temperature in the dark. Subsequently, the protein sample was diluted by adding $100 \mathrm{mM}$ TEAB(Tetraethyl ammonium bromide) to urea $<2 \mathrm{M}$. Finally, trypsin was added at 1:50 trypsin-to-protein mass ratio for the first digestion overnight and 1:100 trypsin-to-protein mass ratio for 4h digestion.

\subsection{TMT/iTRAQ labeling}

After trypsin digestion, the peptide was desalted by Strata X C18 SPE column, vacuum-dried, reconstituted in 0.5 M TEAB, and processed according to the manufacturer's protocol for TMT kit/iTRAQ kit (Sigma, USA). Briefly, $1 \mathrm{U}$ of TMT/iTRAQ reagent was thawed and reconstituted in acetonitrile. The peptide mixtures were incubated for $2 \mathrm{~h}$ at room temperature and pooled, desalted, and dried by vacuum centrifugation.

\subsection{High-performance liquid chromatography (HPLC) fractionation}

The tryptic peptides were fractionated by high pH reverse-phase HPLC using Thermo Betasil C18 column (5 $\mu \mathrm{m}$ particles, $10 \mathrm{~mm}$ ID (Identity document), $250 \mathrm{~mm}$ length). Briefly, the peptides were first separated with a gradient of $8-32 \%$ acetonitrile ( $\mathrm{pH} 9.0$ ) into 60 fractions over 60 min and then combined into 6 fractions and dried by vacuum centrifugation.

\subsection{IMAC-enriched phosphorylated peptide}

The peptides were dissolved in the enrichment buffer solution ( $50 \%$ acetonitrile/ $6 \%$ trifluoroacetic acid), the supernatant was transferred to the pre-washed IMAC material (AbD Serotec, UK), and incubated for xxx h. Then, a buffer solution consisting of $50 \%$ acetonitrile, $6 \%$ trifluoroacetic acid, and $30 \%$ acetonitrile/ $0.1 \%$ trifluoroacetic acid was used for washing the resin. Finally, $10 \%$ ammonia water was used to elute the modified peptide section, and the eluent was collected and vacuum-frozen. After draining, desalination was carried out according to C18 ZipTips kit (Millipore, Bedford, USA).

\subsection{HPLC/MS/MS analysis}

The purified peptide was solubilized in liquid chromatography mobile phase A ( $0.1 \%$ formic acid solution) and then separated by easy-NLC 1000 ULTRA high-performance liquid phase system. Mobile phase $A$ was an aqueous solution containing $0.1 \%$ formic acid and $2 \%$ acetonitrile, while mobile phase $B$ was an aqueous solution containing $0.1 \%$ formic acid and $90 \%$ acetonitrile. Liquid phase gradient settings were as follows: $0-40$ min, 4-22\% B; $52 \mathrm{~min}, 22-35 \%$ B; 52-56 min, 35-80\% B; 56-60 min, 80\% B, flow rate maintained at $400 \mathrm{~nL} / \mathrm{min}$. The eluted peptides were separated by an ULTRA high-performance liquid system, injected into an NSI ion source for ionization, and then analyzed by Q Exactive Plus mass spectrometry. The ion source voltage was set at $2.0 \mathrm{kV}$, and the peptide fragments and secondary fragments were detected and analyzed using high-resolution Orbitrap. The level of mass spectrum scanning range was set to $350-1800 \mathrm{~m} / \mathrm{z}$, scanning resolution was set to 70,000 , the scanning range of the secondary mass spectrometry was fixed at $100 \mathrm{~m} / \mathrm{z}$, and the resolution of the secondary scanning was set at 35,000 . $A$ data-dependent acquisition (DDA) program was used to select the first 10 peptide parent ions with the highest signal intensity into the HCD collision pool after the first level scan; $31 \%$ energy was used for fragmentation. Secondary mass spectrometry was performed sequentially to improve the effective utilization rate of mass spectrometry. The automatic gain control (AGC) was set at 1E5, the signal threshold was set at 20000 ion/S, the maximum injection time was set at $100 \mathrm{~ms}$, and the dynamic elimination time of tandem mass spectrometry scanning was set at $30 \mathrm{~s}$ to avoid repeated scan of the parent ion.

\subsection{Bioinformatics analysis of phosphorylated proteins}


The original mass spectrometry data were retrieved using the database Maxquant (V1.5.2.8, China). The retrieval parameter settings were as follows: Swissprot_Human (20422 sequences) was used as the database, and the negative library was added to calculate the positive error rate (FER) caused by random matching to eliminate the influence of contaminated proteins in identification results. The enzyme digestion method was set as trypsin/P, the maximum number of leakage sites of the enzyme was set as 2 , the minimum length of the peptide segment was set as 7 amino acid residues, and the maximum number of modifications of the peptide segment was set as 5 . The mass error tolerance of the primary parent ion of the first search and main search is set as $20 \times 10^{-6}$ and $5 \times 10^{-6}$, respectively, and the mass error tolerance of the secondary fragment ion is $0.02 \mathrm{Da}$. The alkylation of cysteine was fixed, while the variable modification was methionine oxidation, $\mathrm{n}$-terminal acetylation, deamidation (NQ), and phosphorylation of serine, threonine, and tyrosine. The quantitative method was set as TMT-6plex, and the Protein identification and PSM(Anti-PSM/Proteasome 20S) identification false discovery rate (FDR) was set at 1\%. The differences between phosphorylated proteins were detected via a volcanic diagram to characterize these genes through the GO (Fisher's exact test), function, KEGG( Kyoto Encyclopedia of Genes and Genomes) pathways, and analysis of the protein structure domain in cell biology and cell and molecular biology function analysis. Furthermore, Clusters of Orthologous Groups of proteins(COG/KOG) database function classification statistics and Cytoscape were employed to build the protein network.

\subsection{Western blotting}

The lung cancer A549 cells were cultured for $24 \mathrm{~h}$ until $80-90 \%$ confluency was achieved. The cells were treated with 9 mmol/L merecidin $6 \mathrm{~h}$ and observed under a light microscope. The cells were shrunk, their nuclear membranes thickened, or shattered and dissolved. An equivalent of 10-20 mg protein was resolved using SDS-PAGE and transferred to PVDF Hybond-p membranes (GE Healthcare, Milano, Italy). Then, the membranes were probed with primary antibodies against ULK1 (1:1,000 dilution in TBST and 5\% skim milk; Abcam, UK), MAPK1 (1:2,000; Abcam), ATG13 (1:1,000; Abcam), and b-tubulin (1:2,000; Abcam) at $4^{\circ} \mathrm{C}$ overnight. Subsequently, the membranes were incubated with peroxidase-conjugated Affinipure goat antirabbit $\mathrm{LgG}(\mathrm{H}+\mathrm{L})(1: 1000)$ secondary antibodies at room temperature for $1 \mathrm{~h}$. The immunoreactive bands were visualized using ECL Advance (GE Healthcare)., and the intensity quantified using Image Lab v3.0 software (Bio-Rad Laboratories Inc., Hercules, CA, USA).

\subsection{Statistical analysis}

All values were expressed as mean \pm standard deviation calculated from three independent experiments. The data were analyzed with a one-way analysis of variance (ANOVA), followed by Tukey's multiple comparison posthoc test (SPSS 24.0 software). $P<0.05$ and $P<0.01$ indicated statistical significance.

\section{Results}

\subsection{Phosphorylation proteomics results}

A total of 10320 phosphorylated modification sites located on 3,089 proteins were identified, among which, 9094 provided quantitative information. The identification data were filtered by 0.75 criterion to identify 8,317 phosphorylation modification sites on 2,900 proteins; of these 7,937 sites for 2,787 proteins contained quantitative information (Fig. $1 \mathrm{~A}$ ). According to the fold-change $>$ or $<1.5,246$ upregulated phosphorylated proteins and 559 downregulated phosphorylated proteins, corresponding to 485 and 860 phosphorylated sites were identified, respectively (Fig. 1B). The distribution of proteins is shown (Fig. 1C) in the volcanic diagram. Red represents an upregulated protein, blue represents downregulated protein, and the difference in protein expression (drug/mock) occurred within 0.3-3 fold.

\subsection{Motif analysis of phosphorylated proteins}

In order to understand the modified proteins identified in the data and the functions and characteristics of the proteins, the software Protein motif was applied to analyze the regularity of amino acid sequences before and after all phosphorylation modification sites in the samples and calculate the regularity trend of amino acid sequences within the region harboring the phosphorylation modification sites. Figure 2 shows the motif enrichment heat map of amino acids upstream and downstream of all identified phosphorylation modification sites; red indicates significant enrichment of the amino acids near the modification site, while green indicates a significant reduction of the amino acid near the modification site. The results showed that the downstream amino acids were significantly enriched at the phosphorylation modification sites. Also, the Gene Ontology (GO) protein annotation, the distribution of biological process, cell composition, and molecular function of proteins corresponding to the differentially modified sites in the GO secondary annotation were analyzed (Fig. 3A). Furthermore, the phosphorylated proteins were distributed in the cell process, biological regulation, cell and organelle composition, molecular binding, and catalytic activity. The prediction and classification statistics of the subcellular structure of the differentially modified proteins are shown in Fig. 3B, indicating that the differentially modified proteins are mainly concentrated in the nucleus and cytoplasm .

\subsection{Functional enrichment analysis of differential phosphorylated proteins}


The functional enrichment of differential proteins is mainly carried out at the three levels of GO classification, KEGG pathway, and protein domain. For the enrichment test, the P-value showed significant enrichment of differentially modified proteins $(P<0.05)$. Differences were detected in the corresponding protein phosphorylation modification sites with respect to the biological process (Fig. 3C), cellular component (Fig. 3D), and molecular function (Fig. 3E). These findings showed significant differences in the degradation of proteins involved in cell signal transduction, DNA transfer and translation of the cellular energy metabolism, protein synthesis, and the formation of the cytoskeleton. Proteins corresponding to the differential phosphorylation modification sites were classified in the protein domain (Fig. 3F). KEGG pathways mainly included metabolism, genetic information processing, environmental information processing, cell processes, human diseases, and drug development. In this study, pathways such as mTOR, ErbB, PI3K/AKT,and AMPK were enriched and monitored (Fig. 3G).

\subsection{Functional classification of different proteins}

The Chinese definition of COG, namely "homologous protein," is compared and analyzed in the database, and the COG/KOG functional classification statistics of the differentially modified proteins were carried out (Fig. 4). It was found that differential phosphorylated proteins mainly focus on cell signal transduction, RNA transcription, translation processing and modification, ribosomes, cytoskeleton proteins, intracellular material transport, protein secretion, and vesicle transport.

\subsection{Protein interaction network analysis}

The protein network (Fig. 5) interaction correlation showed that the whole protein network consisted of HADC1(Fig. 6A), MAPK1, RPL23A, SRSF3H, and NCBP1 as the core interaction network. Among these, the difference is related to apoptosis protein, extracellular signal-regulated kinase activated protein kinase 1 (MAPK1),mitogen-activated protein kinase 3(MAPK3), stathmin 1(STMN1), Ephrin type-B receptor 2(EPHB2). The interaction analysis of apoptosis-related proteins was represented in Fig. 6B. In addition, screening revealed significantly differentially expressed proteins (Table 1): autophagy-related protein 2 homolog B (ATG2B), autophagy-related protein homolog 13 (ATG13), serine/threonineprotein kinase (ULK1), Ras-related protein Rab-7a (RAB7A), and autophagy-related protein 9A (ATG9A), that were markedly upregulated (Fig. 7), while (mitogen-activated protein kinase 1 (MAPK1), eukaryotic translation initiation factor 4B (EIF4B), protein EGFR, tuberin growth factor receptor (TSC2), and serine/threonine-protein kinase 4 (PAK4), and alpha serine/threonine-protein kinase (AKT1) were significantly downregulated.

\section{Discussion}

Protein composition is the giant arm of the post-genetic age, and many researchers have investigated the pathogenesis of cancer through proteomics analysis. A previous study ${ }^{40}$ demonstrated that all the proteins might be expressed in the tumor cells and tumor microenvironment. Surprisingly, cluster analysis revealed that diffuse gastric cancer could not only be divided into three molecular subtypes (PX1-3), but also was closely related to the survival prognosis and chemotherapy sensitivity. Sato et al. ${ }^{41}$ found that the use of CKAP4 as a biomarker changes the current treatment of lung cancer patients. In addition, the combination of CKAP4 and conventional markers can significantly improve diagnostic accuracy.

Although there have been extensive studies on LL-37, the phosphorylation analysis of LL-37 and its derived peptides in cells has not yet been reported. In this study, we implemented a quantitative proteomics analysis to determine how protein phosphorylation systematically advances apoptosis of lung cancer A549 cells treated with antimicrobial peptide merecidin.

Next, we used to quantitative proteomics research strategy via TMT labeling, phosphorylation modification enrichment techniques, and highresolution liquid chromatography-mass spectrometry. The localization probability was $>0.75$. The data were filtered to identify 8,317 phosphorylation sites on 2,900 proteins, of which 7,937 sites for 2,787 proteins provided quantitative information. We identified that 485 sites in the drug group vs mock comparison group were upregulated, and 860 sites were downregulated. The identified proteins were annotated regarding GO, protein domains, KEGG pathways, and subcellular structure localization to describe the biological process, cellular component, and molecular function. The protein motif is closely related to the biological functions of proteins, which was used to analyze the regularity of amino acid sequences before and after all phosphorylation modification sites in the sample. The data suggested that in the biological process, the protein motif is mainly involved in cell process, biological regulation, and signal transduction. The cell composition mainly involves the cytoplasm, cell membrane, and organelle, and the molecular function involves protein molecule binding, activation, and molecular function regulation. In terms of subcellular composition, the composition of the nucleus, organelles, cytoplasm, and mitochondria are involved. The functional enrichment results showed that differentially expressed proteins are involved in the regulation of the whole process from DNA replication to RNA transcription and translation, as well as protein-peptide chain formation, plait, and domain formation. The enrichment of the KEGG pathway revealed the proteins, such as GnRH, RIG-I-like, Ras, ErbB, PI3K-Akt, mTOR, and AMPK in signaling pathways. In summary, merecidin-treated A549 cells affect the cell functions from the nucleus to the cytoplasm and organelles. In addition, the COG/KOG functional classification statistics identified cytoskeleton proteins, intracellular material transport, secretion, and vesicle transport. 
Historically, we found that the antimicrobial peptide merecidin induced apoptosis in lung cancer A549 cells ${ }^{34,35}$. We also screened an array of proteins from the identification protein interaction network (Fig. 5) as follows: MAPK1, also known as ERK2, is associated with tumor proliferation $^{42}$, differentiation, and invasion, and its upregulation is associated with the occurrence and development of various tumors $^{43}$.Mitogen-activated protein kinase 3(MAPK3),knows as ERK1,whereas ERK pathway mediates cellular survival and growth,as well as leads to increased apoptosis ${ }^{44} \square$ the study suggests that MAPK3 has close relationship with cardiomyocyte apoptosis in IRI ${ }^{45}$. MAPK14 gene, known as p38a, is one of the four family members of MAP kinase which exert versatile functions in diverse cellular processes in cancer: cell cycle regulation, proliferation, survival and motility ${ }^{46}$,as well as MAPK14 (P38) can be involved in a variety of cellular stresses, including internal metabolic stress, DNA damage, external growth factor pathway, cell-matrix interaction and intercellular communication 47,48 .Mesquita et al have showed p38a inhibition Cellular migration was impaired and decreased the gastric cancer cell proliferation by provoking cell cycle arrest and cell death ${ }^{49}$.In this study, the phosphorylation site T180/Y182 was downregulated by merecidin .Ephrin type-B receptor 2(EPHB2) is associated with the occurrence and development of A variety of diseases.EPHB2 has been shown to be associated with cells apoptosis in many studies ${ }^{50,51}$. ${ }^{52}$ Dong et al have suggested that EphB/ephrinB reverse signaling is involved in retinal ganglion cell (RGC) apoptosis in experimental glaucoma.In our study ,the phosphorylation site S776/S575of EphB2 Upregulated. There are also a number of identified apoptosis-related proteins that have been published in the previous by our research group ${ }^{53}$.Furthermore, we speculated that merecidin induction of A540 cells enriched the KEGG-identified classic apoptosis-related signaling pathways: ErbB ${ }^{54}, \mathrm{PI} 3 \mathrm{~K} / \mathrm{AKT}^{55}$, and $\mathrm{AMPK}^{-\mathrm{Akt}^{56}}$.

We suspected that merecidin might induce autophagy of A549 cells. Based on the literature, we selected the following proteins from the protein interaction network. ATG2B was mainly involved in the formation of autophagosomes and fused with lysosomes before degradation ${ }^{57}$. ATG2A and ATG2B genes are involved in autophagosome formation in HeLa cells ${ }^{58}$. In this study, the phosphorylation was upregulated at sites S240/S255 of ATG2B. ATG9A is involved in autophagosome and cytoplasmic vacuolar transport (Cvt) and is the only transmembrane protein. Atg9 plays a crucial role in the formation of autophagosomes ${ }^{59}$. After the treatment of A549 cells with merecidin, the phosphorylation at site S656/738/735 is upregulated. ATG13 ${ }^{60}$ is involved in autophagy and targets the mTOR kinase signaling pathways by controlling the ATG13ULK1 phosphorylation status and ATG13-ULK1 to regulate autophagy. Human serine/threonine protein kinase ULK1 is one of the major regulatory factors of apoptosis. ULK1/2 induces proliferation ${ }^{61}$, autophagy in ULK complex, and controls autophagosome biogenesis via early signaling pathways ${ }^{62}$. The S738/S735 of ATG13 and S479/S477 of ULK1 was upregulated in this study. RAB7A is a key regulator of lysosomal transport and critical for autophagosome-lysosome fusion ${ }^{63}$. As a vital protein in the F0X01/RAB7 signaling pathway, RAB7A induces the autophagy by regulating RAB7 upregulation. The phosphorylation at S72 site in RAB7 is also upregulated in this study. MAPK $1^{64}$ harbors several signal integration points that participate in a variety of cellular processes, such as proliferation, differentiation, transcription, and development. The upstream kinase phosphorylation activation MAPK1/ERK2 was applied for the stimulation of the nucleus, and the inhibition of phosphorylation of MAPK1 activates autophagy 65,66 . The phosphorylation site T185/Y187 of MAPK1 is upregulated after treatment with merecidin. EIF4B is also known as cap-binding protein, one of the vital components of the elF4F complex. The high expression can lead to tumor proliferation, invasion, and metastasis. Zhang et al. ${ }^{67}$ found that inhibition of EIF4B expression enhances autophagic death, and S579 of EIF4B was found to be upregulated in this study. The EGFR signaling pathway regulates the autophagy process; inhibition of the expression of EGFR elevates autophagy, leading to autophagic death ${ }^{68}$. In this study, TSC2 was found to activate autophagy by activating the TSC2/mTOR signaling pathway $^{69,70}$, and the phosphorylation site S1132/S1452 of TSC2 is downregulated. PAK4 is highly expressed in a variety of tumor tissues and cells and is related to cytological behaviors, such as cell proliferation, cycle, and migration. Low expression of PAK4 can upregulate p53, inhibit mTOR or Akt/mTOR signaling pathway, inhibits cell migration, and activate autophagy ${ }^{71}$, or the signaling pathway ${ }^{72}$ can be downregulated, thus activating autophagy ${ }^{73,74}$. The phosphorylation site S103 of PAK4 downregulated after merecidin treatment. AKT1 regulates metabolism, proliferation, cell survival, growth, and angiogenesis, inhibits growth, and induces apoptosis of non-SCLC by directly regulating Akt1/2 ${ }^{29}$, mediated by phosphorylation of serine and/or threonine by a series of downstream substrates. In addition, Akt1/Foxo3a ${ }^{75}$ and $\mathrm{PI3K} / \mathrm{AKT} / \mathrm{mTOR}^{76}$ signaling pathways are associated with autophagy, and the phosphorylation at site S124 of AKT1 is downregulated after merecidin treatment. Additionally, we found that the autophagy-related pathways mTOR and PI3K-Akt were selected from KEEG pathway enrichment data. In conclusion, we hypothesized that merecidin induces autophagy in A549 cells and by mTOR and/or PI3K-Akt signaling pathway.

\section{Conclusion}

In summary, merecidin treatment of lung cancer A549 cells induced apoptosis through signaling pathways such as ErbB, PI3K/AKT, and AMPKAkt. In addition, the antimicrobial peptide merecidin might induce autophagy in A549 cells. This study laid a solid foundation for further exploration of merecidin in lung cancer A549 cells.

\section{Abbreviations}

TMT: Tandem Mass Tags 
iTRAQ : isobaric tags for $r$ elative and absolute quantitation

LC-MS/MS : liquid chromatograph-mass spectrometer

HPLC : High-performance liquid chromatography

IMAC : Immobilized Metal Ion Affinity Chromatography

GO\Gene Ontology

COG: Clusters of Orthologous Groups

TSA NAM: Trichostatin A

HCD : Suramin sodium salt

KEGG $₫$ Kyoto Encyclopedia of Genes and Genomes

PSMIProteasome

\section{Declarations}

\section{Ethics approval and consent to participate}

Not applicable.

\section{Consent for publication}

Not applicable.

\section{Availability of data and material}

Tables,figures,covering letter,Highlights and supplement are included within the additional files.STRING: functional protein association networks (https://string-db.org/cgi/input?sessionld=bN158vsr

WaZz\&input_page_show_search=on)

\section{Competing interests}

The authors declare no conflict of interest.

\section{Funding}

This study was supported by the National Natural Science Foundation of China (No.81760661, No. 81560573) but did not receive any specific grant from the funding agencies in the public, commercial, or not-for-profit sectors.

\section{Authors' contributions}

1. Jia Qinqin is the writer, contributor of this paper and executor of the experiment of this study.

2. Zhang qiannan, Yang Tingting is the executor of the experiment.

3. Song jinxuan and Wang Yarong were mainly experimental assistants.

4. Wang xiuqing is the main person in charge of this experiment, participating in the experiment, guiding the article, and corresponding author of this paper.

\section{Acknowledgements}

We thank Professor Guangshun Wang of the Nebraska Medical Center in the United States for the design and synthesis of merecidin. We also thanks the Affiliated Hospital of Ningxia Medical University (Department of Stem cell institute) for the kind human lung adenocarcinoma cancer cell line A549.

\section{References}


1. Freddie, Bray, Jacques,et al. Global cancer statistics 2018: GLOBOCAN estimates of incidence and mortality worldwide for 36 cancers in 185 countries.[J]. CA: a cancer journal for clinicians, 2018.

2. Hoffman, P. C.; Mauer, A. M.; Vokes, E. E. J. L., Lung cancer. 2000, 355 (9202), 479-485.

3. Y. Nilssen, T.E. Strand, L. Fjellbirkeland,et al. Lung cancer survival in Norway, 1997-2011: from nihilism to optimism, Eur. Respir. J. 47 (2016) 275-287.

4. Rafael Meza,Clare Meernik,Jihyoun Jeon,et al. Lung cancer incidence trends by gender, race and histology in the United States, $1973-2010$. [J]. PLoS ONE,2017,10(3).

5. M. Grivaux, B. Duvert, P. Ferrer-Lopez, et al. One-year survival improvement in lung cancer in France. Results of the prospective real life studies KBP-2000-CPHG and KBP-2010-CPHG, Rev. Pneumol. Clin. 72 (2016) 163-170.

6. Isabelle Finke,Gundula Behrens,Lars Schwettmann,et al. Socioeconomic differences and lung cancer survival in Germany: Investigation based on population-based clinical cancer registration[J]. Lung Cancer,2020,142.

7. Claudia Allemani,Tomohiro Matsuda,Veronica Di Carlo,et al,D. Global surveillance of trends in cancer survival 2000-14 (CONCORD-3): analysis of individual records for 37513025 patients diagnosed with one of 18 cancers from 322 population-based registries in 71 countries[J]. The Lancet,2018,391(10125).

8. Ismail Hamid D,Jones Ahoi,Kim Jung H,et al. RF-Phos: A Novel General Phosphorylation Site Prediction Tool Based on Random Forest.[J]. BioMed research international,2016,2016.

9. Min-Sik Kim,Sneha M. Pinto,Derese Getnet,et al. A draft map of the human proteome[J]. Nature: International weekly journal of science,2014,509(7502).

10. [1]Fiona M. Menzies,Angeleen Fleming,David C. Rubinsztein. Compromised autophagy and neurodegenerative diseases[J]. Nature Reviews Neuroscience,2015,16(6).

11. [1]Lei-Lei Chen,Yong-Bo Wang,Ju-Xian Song,et al. Phosphoproteome-based kinase activity profiling reveals the critical role of MAP2K2 and PLK1 in neuronal autophagy[J]. Autophagy,2017,13(11).

12. David Ochoa,Andrew F. Jarnuczak,Cristina Viéitez,et al. The functional landscape of the human phosphoproteome[J]. Nature Biotechnology: The Science and Business of Biotechnology,2020,38(3).

13. David J. Clark,Saravana M. Dhanasekaran,Francesca Petralia,et al. Integrated Proteogenomic Characterization of Clear Cell Renal Cell Carcinoma[J]. Cell,2020,180(1).

14. Di Zhang,Zhanyun Tang,He Huang,et al. Metabolic regulation of gene expression by histone lactylation[J]. Nature: International weekly journal of science,2019,574(D1).

15. Suhas Vasaikar,Chen Huang,Xiaojing Wang,et al. Proteogenomic Analysis of Human Colon Cancer Reveals New Therapeutic Opportunities[J]. Cell,2019,177(4).

16. Frohm M,Agerberth B,Ahangari G,et al. The expression of the gene coding for the antibacterial peptide LL-37 is induced in human keratinocytes during inflammatory disorders.[J]. The Journal of biological chemistry,1997,272(24).

17. Murakami, M.; Ohtake, T.; Dorschner, R. A., et al., Cathelicidin antimicrobial peptides are expressed in salivary glands and saliva. J Dent Res 2002, 81 (12), 845-50.

18. Hase, K.; Murakami, M.; limura, M., et al., Expression of LL-37 by human gastric epithelial cells as a potential host defense mechanism against Helicobacter pylori. 2003, $125(6), 1613-1625$.

19. Bucki Robert,Leszczyńska Katarzyna,Namiot Andrzej,et al. Cathelicidin LL-37: a multitask antimicrobial peptide.[J]. Archivum immunologiae et therapiae experimentalis,2010,58(1).

20. Wang Guangshun,Narayana Jayaram Lakshmaiah,Mishra Biswajit,et al. Design of Antimicrobial Peptides: Progress Made with Human Cathelicidin LL-37.[J]. Advances in experimental medicine and biology,2019,1117. Adv Exp Med Bio/ 2019, 1117, $215-240$.

21. da Silva Bruno Rocha,de Freitas Victor Aragão Abreu,Nascimento-Neto Luiz Gonzaga, et al. Antimicrobial peptide control of pathogenic microorganisms of the oral cavity: a review of the literature.[J]. Peptides,2012,36(2).

22. Zasloff Michael. Antimicrobial peptides of multicellular organisms.[J]. Nature,2002,415(6870).

23. Chang-Chun Lee,Yen Sun,Shuo Qian,et al. Huang. Transmembrane Pores Formed by Human Antimicrobial Peptide LL-37[J]. Biophysical Journal,2011,100(7).

24. Johansson J . Conformation-dependent Antibacterial Activity of the Naturally Occurring Human Peptide LL-37[J]. Journal of Biological Chemistry, 1998, 273(6):3718-3724.

25. Mishra Biswajit,Wang Guangshun. Individual and Combined Effects of Engineered Peptides and Antibiotics on Pseudomonas aeruginosa Biofilms.[J]. Pharmaceuticals (Basel, Switzerland),2017,10(3).

26. Biswajit Mishra,Guangshun Wang. Titanium surfaces immobilized with the major antimicrobial fragment FK-16 of human cathelicidin LL-37 are potent against multiple antibiotic-resistant bacteria[J]. Biofouling,2017,33(7). 2020. 
27. Ren Shun X,Cheng Alfred S L,To Ka F,et al. Host immune defense peptide LL-37 activates caspase-independent apoptosis and suppresses colon cancer.[J]. Cancer research,2012,72(24).

28. Wu William Ka Kei,Sung Joseph Jao Yiu,To Ka Fai,Yu Le,et al. The host defense peptide LL-37 activates the tumor-suppressing bone morphogenetic protein signaling via inhibition of proteasome in gastric cancer cells.[J]. Journal of cellular physiology,2010,223(1).

29. Li-Li An,Xiao-Tong Ma,Ying-Hua Yang,et al. Marked Reduction of LL-37/hCAP-18, an Antimicrobial Peptide, in Patients with Acute Myeloid Leukemia[J]. International Journal of Hematology,2005,81(1).

30. Ying-Hua Yang,Guo-Guang Zheng,Ge Li,et al. Expression of LL-37/hCAP-18 gene in human leukemia cells[J]. Leukemia Research,2003,27(10).

31. M. Kittaka, H. Shiba, M. Kajiya,et al. Antimicrobial peptide LL37 promotes vascular endothelial growth factor-A expression in human periodontal ligament cells[J]. Journal of Periodontal Research, 2013.

32. Yamasaki Kenshi,Schauber Jürgen,Coda Alvin,et al. Kallikrein-mediated proteolysis regulates the antimicrobial effects of cathelicidins in skin.[J]. FASEB journal : official publication of the Federation of American Societies for Experimental Biology,2006,20(12).

33. Li Jun,Hui Liyuan ,Zhan Shisheng,et al.Inhibitory effect of antimicrobial peptide 17BIPHE2 on human lung adenocarcinoma Cell Line A549[J].Cancer prevention research,2017,44(10):659-664. Cell 176 (1-2), 11-42.

34. Li Jun . Inhibitory effect of antimicrobial peptide 17BIPHE2 on lung adenocarcinoma A549 cells and its mechanism[D].Ningxia Medical University,2018.

35. Zhan Shisheng,Li Jun ,Yang Tingting ,et al.Merecidin induces apoptosis of human lung adenocarcinoma cell line A549 [J]. Basic Medicine and clinical Practice,2019,39(04):473-477.

36. Hui Liyuan . Antimicrobial peptide 17BIPHE2 inhibits biofilm of Staphylococcus aureus and Pseudomonas aeruginosa [D]. Ningxia Medical University,2017.

37. Wang Yarong,Zhu Mingxing ,Zhang Fan ,et al.Effect of phosphodiesterase PA4781 of C-DI-GMP on inhibition of pseudomonas aeruginosa biofilm by antimicrobial peptide Merecidin [J]. Bulletin of Microbiology,2020,47(03):868-879.

38. Wang Guangshun,Hanke Mark L,Mishra Biswajit,et al. Transformation of human cathelicidin LL-37 into selective, stable, and potent antimicrobial compounds.[J]. ACS chemical biology,2014,9(9).

39. Xiuqing Wang,Biswajit Mishra,Tamara Lushnikova,et al. Amino Acid Composition Determines Peptide Activity Spectrum and Hot-Spot-Based Design of Merecidin[J]. Advanced Biosystems,2018,2(5).

40. Sai Ge,Xia Xia,Chen Ding,et al. A proteomic landscape of diffuse-type gastric cancer[J]. Nature Communications,2018,9(1). (1), 1012.

41. Ueda Koji,Katagiri Toyomasa,Shimada Takashi,et al. Comparative profiling of serum glycoproteome by sequential purification of glycoproteins and 2-nitrobenzensulfenyl (NBS) stable isotope labeling: a new approach for the novel biomarker discovery for cancer.[J]. Journal of proteome research,2007,6(9).

42. Xu M , Li J , Wang X, et al. MiR-22 suppresses epithelial-mesenchymal transition in bladder cancer by inhibiting Snail and MAPK1/Slug/vimentin feedback loop[J]. Cell Death \& Disease, 2018, 9(2):209.

43. Usman M , Tanveer F , llyas A, et al. Varlitinib Mediates Its Activity Through Down Regulating MAPK/EGFR Pathway in Oral Cancer[J]. Current Proteomics, 2020, 17(1):51-58.

44. Liu J , Mao W , Ding B, et al. ERKs/p53 signal transduction pathway is involved in doxorubicin-induced apoptosis in H9c2 cells and cardiomyocytes[J]. American journal of physiology. Heart and circulatory physiology, 2008, 295(5):H1956-65.

45. Liu, Yaling, Yang,et al. MicroRNA-15b deteriorates hypoxia/reoxygenation-induced cardiomyocyte apoptosis by downregulating Bcl-2 and MAPK3[J]. Journal of Investigative Medicine, 2018.

46. Thamodaran V , Bruce A W . p38 (Mapk14/11) occupies a regulatory node governing entry into primitive endoderm differentiation during preimplantation mouse embryo development[J]. Open Biology, 2016, 6(9):160190.

47. Xu M , Ren Z , Wang X, et al. ErbB2 and p38y MAPK mediate alcohol-induced increase in breast cancer stem cells and metastasis[J]. Molecular Cancer, 2016, 15(1):52.

48. Yang, S.-H, Sharrocks,et al. MAP kinase signalling cascades and transcriptional regulation[J]. Gene An International Journal Focusing on Gene Cloning \& Gene Structure \& Function, 2013.

49. Mesquita F P , Moreira-Nunes C A, Silva E L D , et al. MAPK14 (p38a) inhibition effects against metastatic gastric cancer cells: A potential biomarker and pharmacological target[J]. Toxicology in Vitro, 2020, 66:104839.

50. Zhao Y , Li Q , Li X Y, et al. Involvement of mGluR I in EphB/ephrinB reverse signaling activation induced retinal ganglion cell apoptosis in a rat chronic hypertension model[J]. Brain Research, 2018:S0006899318300313.

51. Lingdan D , Xianglin C , Long Z , et al. Calcium channels are involved in EphB/ephrinB reverse signalinginduced apoptosis in a rat chronic ocular hypertension model[J]. Molecular Medicine Reports, 2018, 17:2465-2471. 
52. Dong L D , Gao F , Wang X H, et al. GluA2 trafficking is involved in apoptosis of retinal ganglion cells induced by activation of EphB/EphrinB reverse signaling in a rat chronic ocular hypertension model.[J]. Journal of Neuroence the Official Journal of the Society for Neuroence, 2015, 35(13):5409-21.

53. Yang Tingting, ZHAN Shisheng, WANG Yarong, et al. Phosphorylated proteomic analysis of antimicrobial peptide Merecidin in human lung adenocarcinoma A549 cell line [J].Chinese Journal of Tumor Biotherapy, 2020(2):115-122.

54. Xia W , Mullin R J , Keith B R , et al. Anti-tumor activity of GW572016: a dual tyrosine kinase inhibitor blocks EGF activation of EGFR/erbB2 and downstream Erk1/2 and AKT pathways.[J]. Oncogene, 2002, 21(41):6255-6263.

55. Huang Y , Wu D , Fan W . Protection of ginsenoside Rg1 on chondrocyte from IL-1 $\beta$-induced mitochondria-activated apoptosis through $\mathrm{PI3K} /$ Akt signaling[J]. Molecular \& Cellular Biochemistry, 2014, 392(1-2):249-257.

56. JIEUN KIM, YONGWOON KIM, SUNGCHUL PARK,et al. Adiponectin Inhibits Palmitate-Induced Apoptosis via AMPK-AKT and cAMP-PKA Pathway in HUVEC[J]. Diabetes, 2007, 56(Suppl 1):A510.

57. Wei Jiali,Ma Zhongliang,Li Yanli,et al. miR-143 inhibits cell proliferation by targeting autophagy-related 2B in non-small cell lung cancer H1299 cells.[J]. Molecular medicine reports,2015,11(1).

58. Wei, Ma, Li, et al. miR-143 inhibits cell proliferation by targeting autophagy-related 2B in non-small cell lung cancer H1299 cells.[J]. Molecular Medicine Reports, 2015, 11(1):571-576.

59. Tamura H, Shibata M , Koike M , et al. Atg9A protein, an autophagy-related membrane protein, is localized in the neurons of mouse brains. [J]. Journal of Histochemistry \& Cytochemistry, 2010, 58(5):443-453.

60. Sebastian; Alers; Sebastian, et al., ATG13. 2014.

61. Li M , Lindblad J L , Perez E , et al. Autophagy-independent function of Atg1 for apoptosis-induced compensatory proliferation[J]. BMC Biology, 2016, 14(1):70.

62. Maria Z , Ian?G. G. The mammalian ULK1 complex and autophagy initiation[J]. Essays In Biochemistry, 2017, 61(6):585-596.

63. Zhou J , Li G , Zheng Y, et al. A novel autophagy/mitophagy inhibitor liensinine sensitizes breast cancer cells to chemotherapy through DNM1L-mediated mitochondrial fission[J]. Autophagy, 2015:00-00., 00-00.

64. Li Q, Wang Y, Peng W, et al. MicroRNA-101a Regulates Autophagy Phenomenon via the MAPK Pathway to Modulate Alzheimer'sAssociated Pathogenesis[J]. Cell Transplantation, 2019, 28(5):096368971985708.

65. Binbin Ni.Effects of transforming growth factor TGF- 1 on autophagy and apoptosis in rat intervertebral disc cells[D]. 2014. Shanghai Jiao Tong University, 2014.

66. Wang, W. A preliminary study on the mechanism of autophagy induced by glycosylation end products in human periodontal cells. Nanjing Medical University, 2014.

67. Meng Zhang,Yanping Ma. Effects of elF4E on CD138 + autophagy in multiple myeloma [J]. Chinese Journal of Experimental Hematology, 2019(5). 2019.

68. Xinqiao Pang,Review. Research progress of autophagy and EGFR inhibitors in tumor therapy[J]. Journal of Clinical and Pathology, 2016, 36(005):681-689. $036(005), 681-689$.

69. Chunhui Song,Yunxi Ji,Zhigang Gong. Adenylate activated protease mediates myocardial mitochondrial autophagy in diabetic rats and its relationship with TSC2 [J]. Journal of Chongqing Medical University, 2020(3):338-342.

70. Hongyan Huang. C/EBP participates in meTH-induced neurotoxicity through the DDIT4/TSC2/mTOR and Trib3/Parkin/ -SYn signaling pathways[D]. 2019.

71. Zhang X Y. Inhibitory effect of PAK4 expression on HepG2 cell proliferation, autophagy and migration and its mechanism [D].

72. Wei S , Gastroenterology D O . MiR-199a/b-3p inhibits the proliferation of gastric cancer cell line MGC-803 via down-regulating PAK4/MEK/ERK signaling pathway[J]. Modern Digestion \& Intervention, 2018.

73. Yunxiao Zhang. Inhibition of PAK4 expression on HepG2 cell proliferation, autophagy, migration and its mechanism[D].

74. Zeng, B.; Shi, W.; Tan, G. J. B. C., MiR-199a/b-3p inhibits gastric cancer cell proliferation via down-regulating PAK4/MEK/ERK signaling pathway. 2018, $18(1), 34$

75. Shiyun Zhang,Zhenling Duan,Xu Lin,et al.Studies on autophagy and apoptosis induced by cisplatin in Ovarian cancer SKOV3 cells [J]. Advances in Modern Obstetrics and Gynecology, 2011, 20(001):45-47.

76. Zhang, Q.; Sheng, X.; Liu, J., et al., Cisplatin induces autophagy of Ishikawa cells in endometrial carcinoma by inhibiting the $\mathrm{PI3K} / \mathrm{AKT} / \mathrm{mTOR}$ signaling pathway. 2017, (5), 25-30.

\section{Tables}

Tab . 1 Top 15 differentially expressed phosphorylated proteins associated with 
apoptosis and their phosphorylation sites after antimicrobial peptide merecidin treatment

\begin{tabular}{|c|c|c|}
\hline \multirow{2}{*}{$\begin{array}{ll}\text { Gene } & \text { Protein description } \\
\text { RB1 } & \text { Retinoblastoma-associated protein }\end{array}$} & \multicolumn{2}{|c|}{ regulatid\$PYPhospho-sites count } \\
\hline & Down & $84012(\mathrm{~S} 811 / \mathrm{S} 212)$ \\
\hline MAPK1Mitogen-activated protein kinase 1 & Down & $0112(\mathrm{~T} 185 / \mathrm{Y} 187)$ \\
\hline ARAF Serine/threonine-protein kinase A-Raf & Down & 3115 (T253) \\
\hline PTK2 Focal adhesion kinase 1 & Down & 2013 (S29/S843/S576) \\
\hline FOXO Forkhead box protein & Down & $0112(\mathrm{~S} 491)$ \\
\hline MARCK\&yristoylated alanine-rich C-kinase substrate & Down & $10112(\mathrm{~S} 27 / \mathrm{S} 167 / \mathrm{S} 163 / \mathrm{T} 150)$ \\
\hline NOTCHReurogenic locus notch homolog protein & Down & $9202(\mathrm{~T} 172 / \mathrm{T} 175)$ \\
\hline ACTINApoptotic chromatin condensation inducer in nucleus & UP & 4004 (S455) \\
\hline EIF5B Eukaryotic translation initiation factor 5B & UP & 5005 (S595/S588/S190) \\
\hline KI67 Proliferation marker protein Ki-67 & Down & 74011 (S538/S374/S648/S357) \\
\hline TP53 Tumor protein p53-inducible protein & Down & $1001(\mathrm{~S} 14)$ \\
\hline AIFM1 Apoptosis-inducing factor 1 & UP & $1001(\mathrm{~S} 254)$ \\
\hline LMNA Prelamin-A & UP & $2002(\mathrm{~S} 107 / \mathrm{S} 301)$ \\
\hline PRKCAProtein kinase C alpha type & UP & 0303 (S226/S249/T497) \\
\hline MAP2KDual specificity mitogen-activated protein kinase kinas & eD2own & 0101 (Т394) \\
\hline BCL-2 Bcl-2-associated transcription factor & Down & 7007 (S315/S397/S264/S259) \\
\hline
\end{tabular}

Tab. 2 Phosphorylated proteins and phosphorylated sites with significant differences after the treatment with antimicrobial peptide mrrecidin

\begin{tabular}{lllllll}
\hline Gene & Protein description & Regulation & PS & PT & PY & Phospho-sites count \\
\hline ATG2B & Autophagy-related protein 2 homolog B & Up & 7 & 0 & 0 & $7 \llbracket S 240 / S 255 \square$ \\
ULK1 & Serine/threonine-protein kinase & Up & 0 & 1 & & $7 \llbracket S 479 / 477 \square$ \\
MAPK1 & Mitogen-activated protein kinase 1 & Down & 0 & 1 & 1 & 2 (T185/Y187) \\
EIF4B & Eukaryotic translation initiation factor 4B & Down & 10 & 0 & 0 & $10 \square S 579 \square$ \\
RAB7A & Ras-related protein Rab-7a & Up & 1 & 0 & 0 & $1 \llbracket S 72 \square$ \\
EGFR & Epidermal growth factor receptor & Down & 11 & 4 & 0 & $15(\mathrm{~S} 104 / 1064 / 1039)$ \\
TSC2 & Tuberin & Down & 5 & 0 & 0 & $5(\mathrm{~S} 1132 / 1452)$ \\
PAK4 & Serine/threonine-protein kinase PAK & Down & 5 & 1 & 0 & $6(\mathrm{~S} 104)$ \\
ATG13 & Autophagy-related protein 13 & UP & 1 & 0 & 0 & $1(\mathrm{~S} 738 / 735)$ \\
ATG9A & Autophagy-related protein 9A & UP & 3 & 0 & 0 & $3(\mathrm{~S} 656 / 738 / 735)$ \\
AKT1 & RAC-alpha serine/threonine-protein kinase & Down & 1 & 0 & 0 & $1(\mathrm{~S} 124)$ \\
\hline
\end{tabular}

\section{Figures}


A

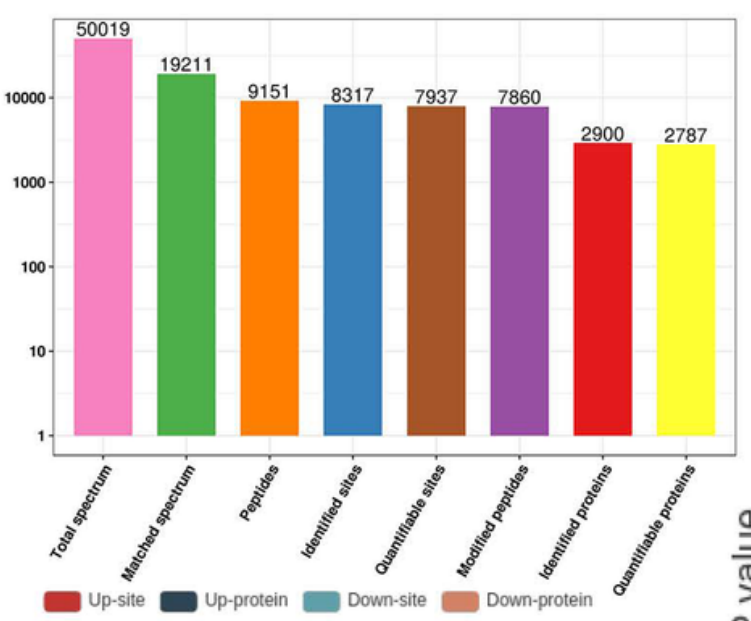

B

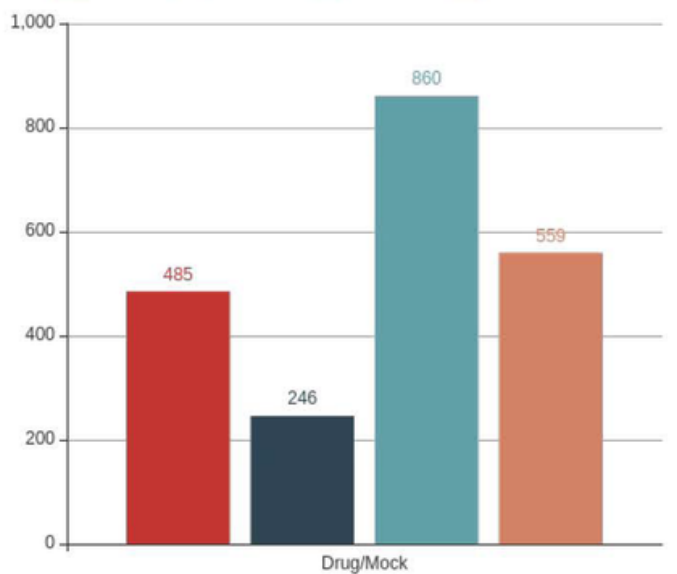

$\mathrm{C}$

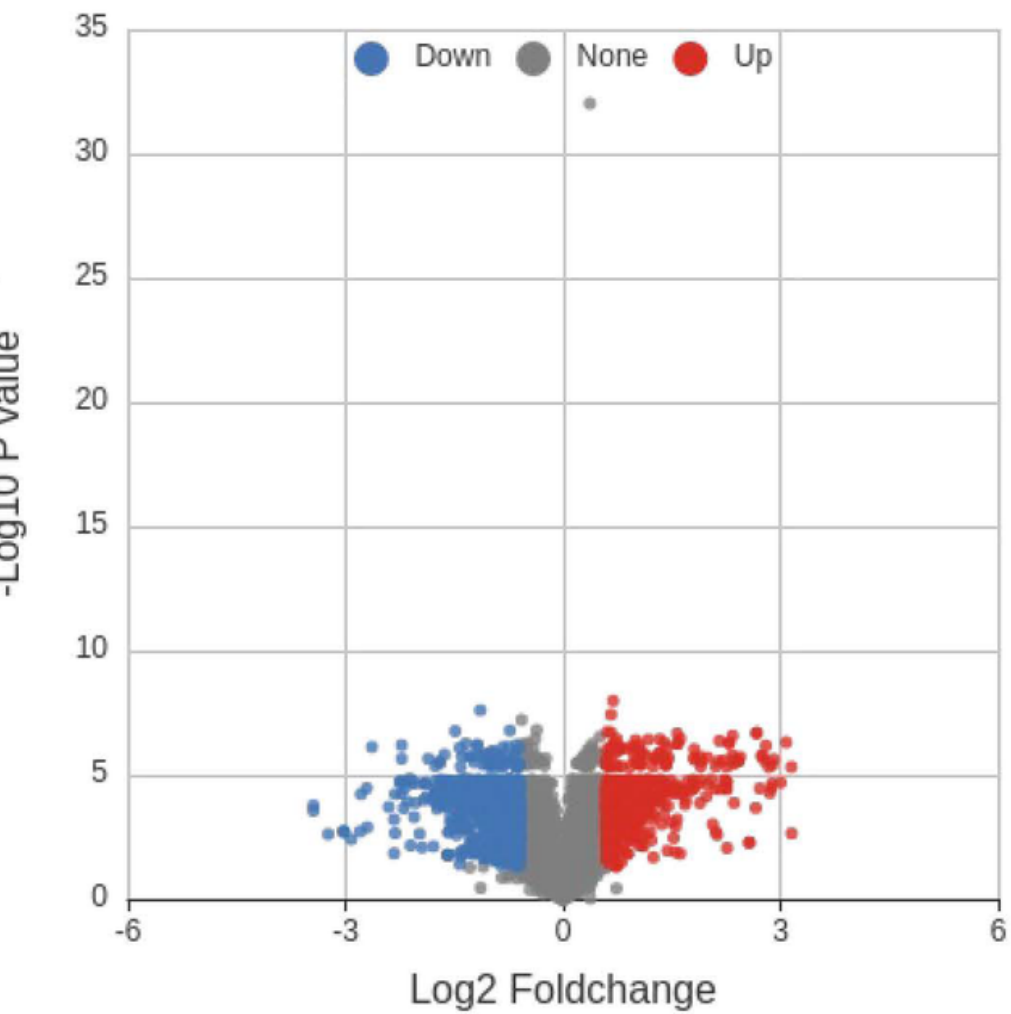

\section{Figure 1}

Phosphorylated proteomics results 1A: Total proteins and corresponding phosphorylation sites were labeled by TMT. 1B: Column diagram of phosphorylated proteins and corresponding phosphorylated sites of A549 cells treated with merecidin; 1C: Volcano map of up-and downregulated differentially phosphorylated proteins. Upregulated proteins are shown in red and downregulated proteins in blue. 


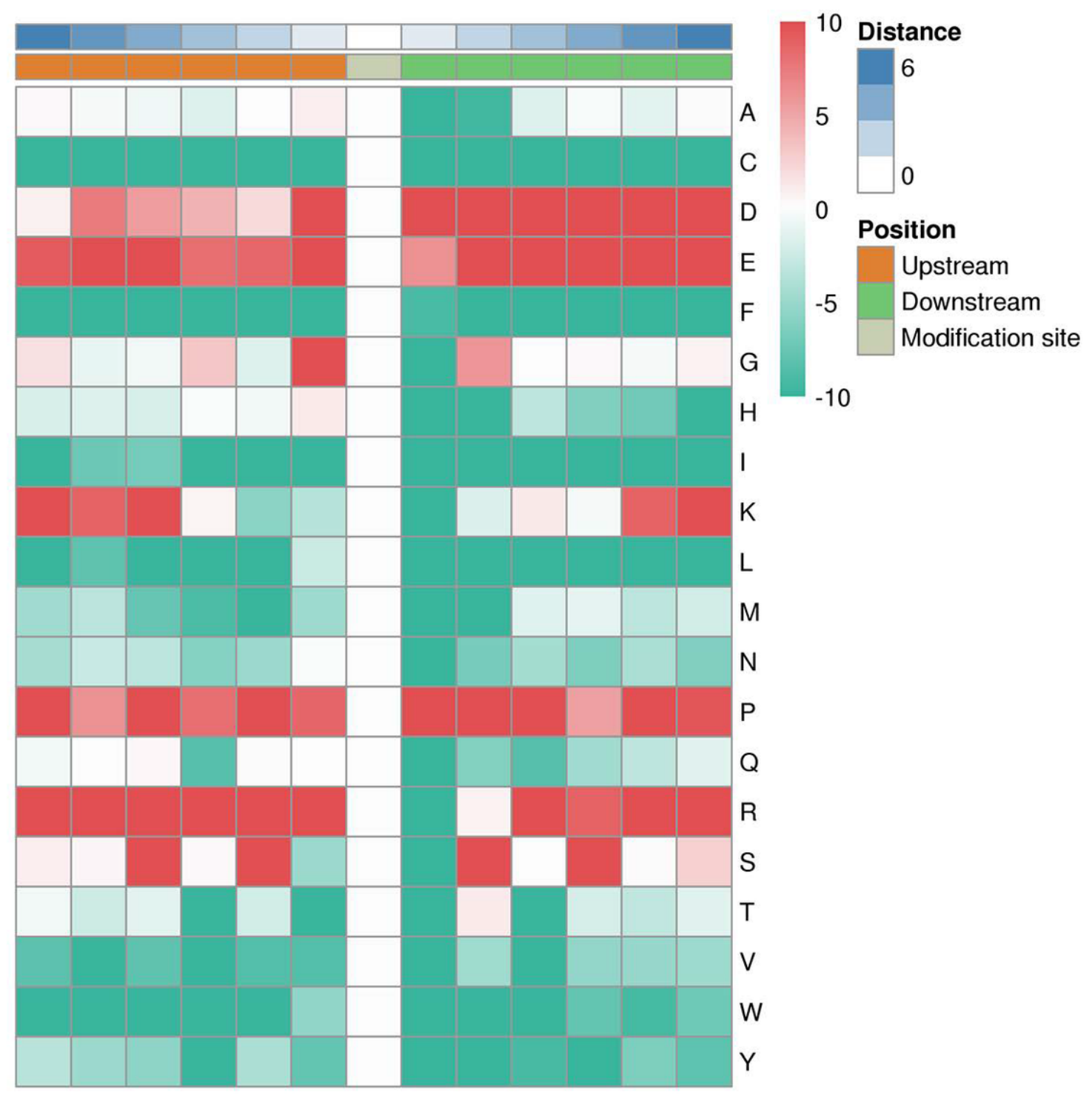

\section{Figure 2}

Motif enrichment heat map of amino acids upstream and downstream of all identified phosphorylation modification sites. Red indicates significant enrichment of the amino acid near the modification site, while green indicates significant reduction of the amino acid near the modification site. 

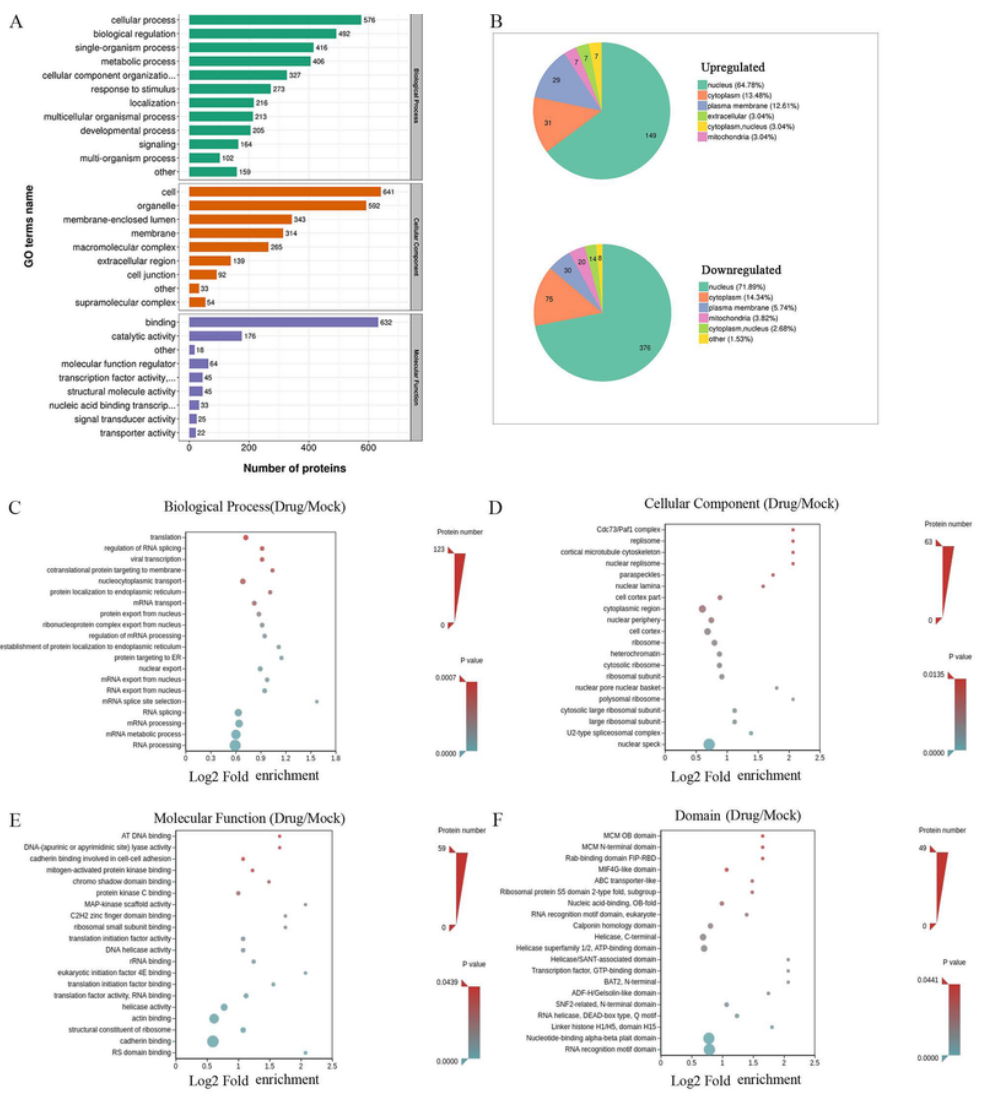

G

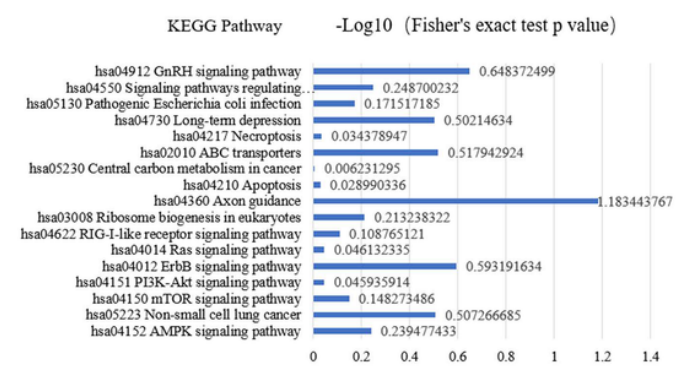

\section{Figure 3}

Functional enrichment analysis of proteins corresponding to differentially modified sites. 3A: Statistical distribution of proteins corresponding to the differential phosphorylation modification sites in GO secondary classification (green represents the distribution of biological processes, red represents the distribution of cell composition, and purple represents the distribution of molecular functions); 3B: Green represents the distribution of biological processes, red represents the distribution of cell composition, and purple represents the distribution of molecular functions; 3C-F: Differential phosphorylation modification site corresponding to protein enrichment distribution bubble map (3C: biological processes; 3D: cell composition; 3E: molecular functions; 3F: domain); 3G: KEGG pathway. 


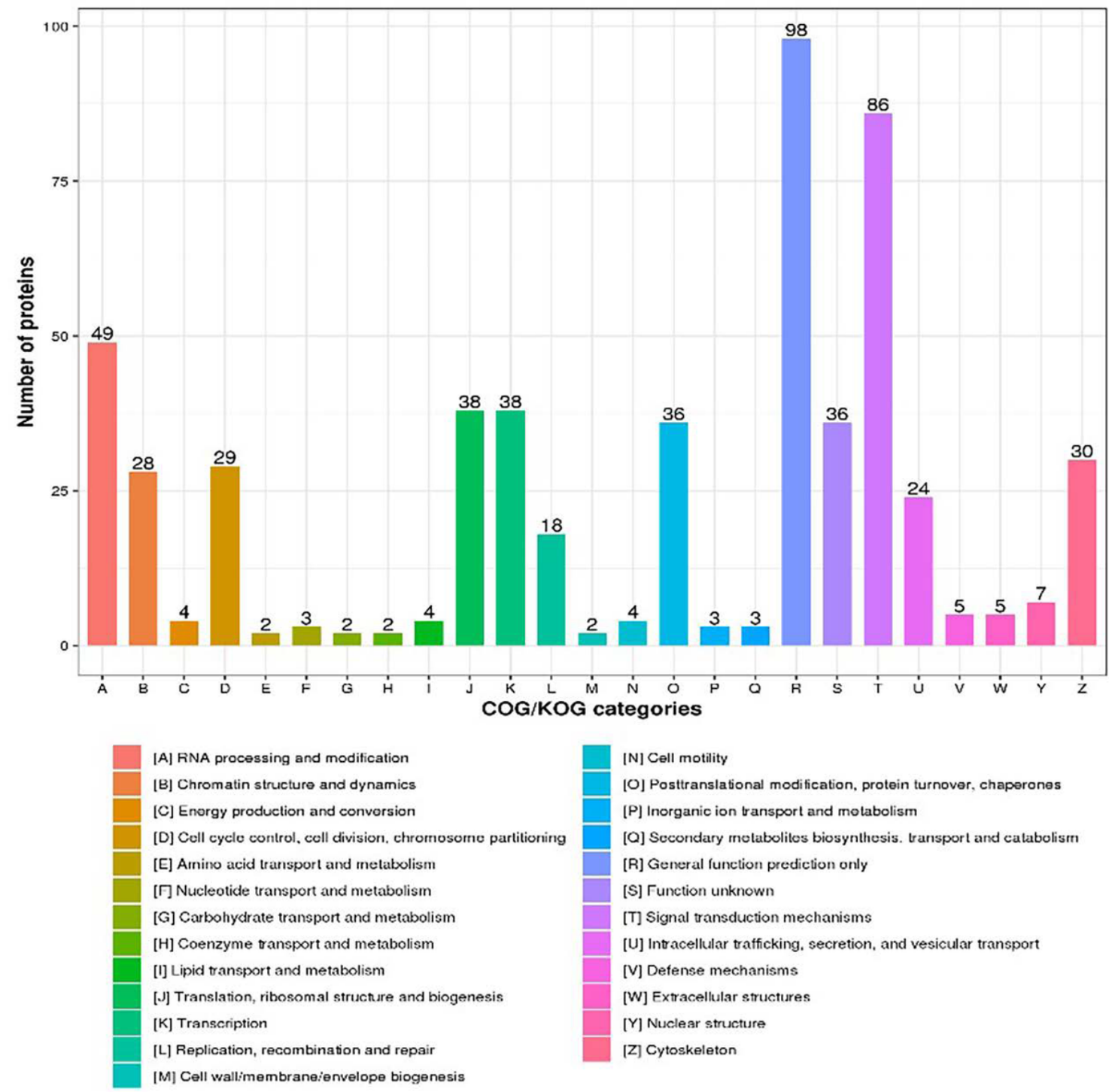

\section{Figure 4}

COG/KOG functional classification distribution map of proteins corresponding to differential phosphorylation modification sites. 


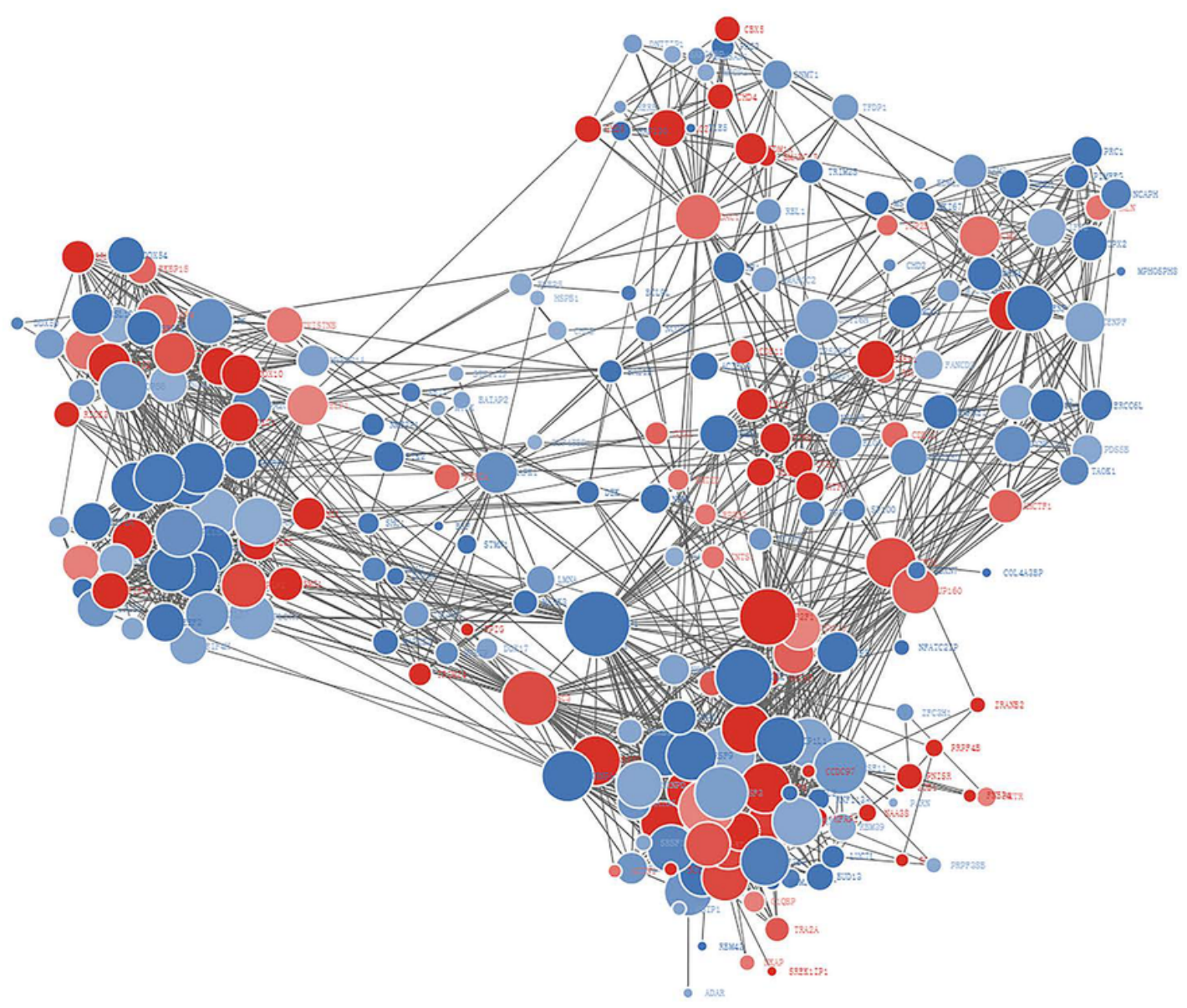

Figure 5

Differential modified protein interaction network diagram.

A

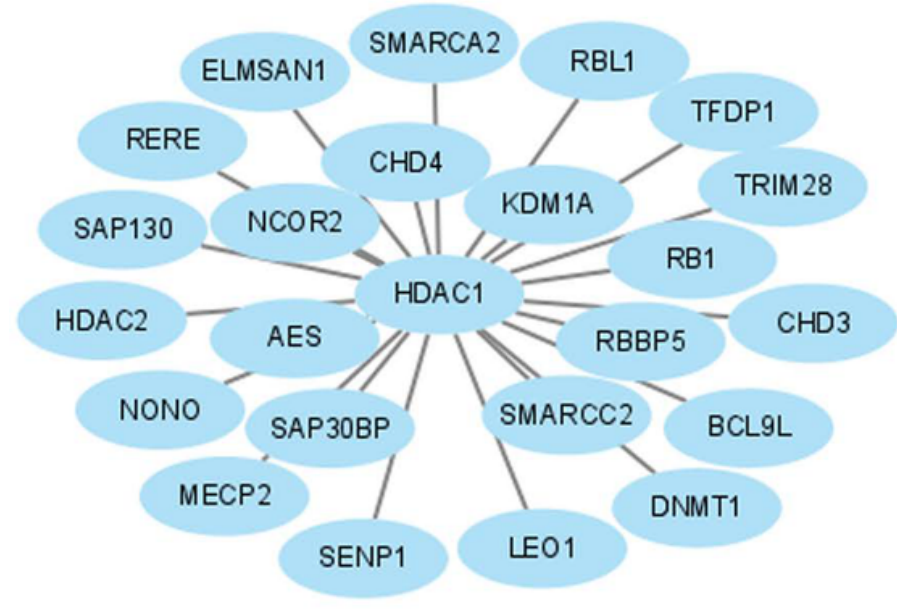

B

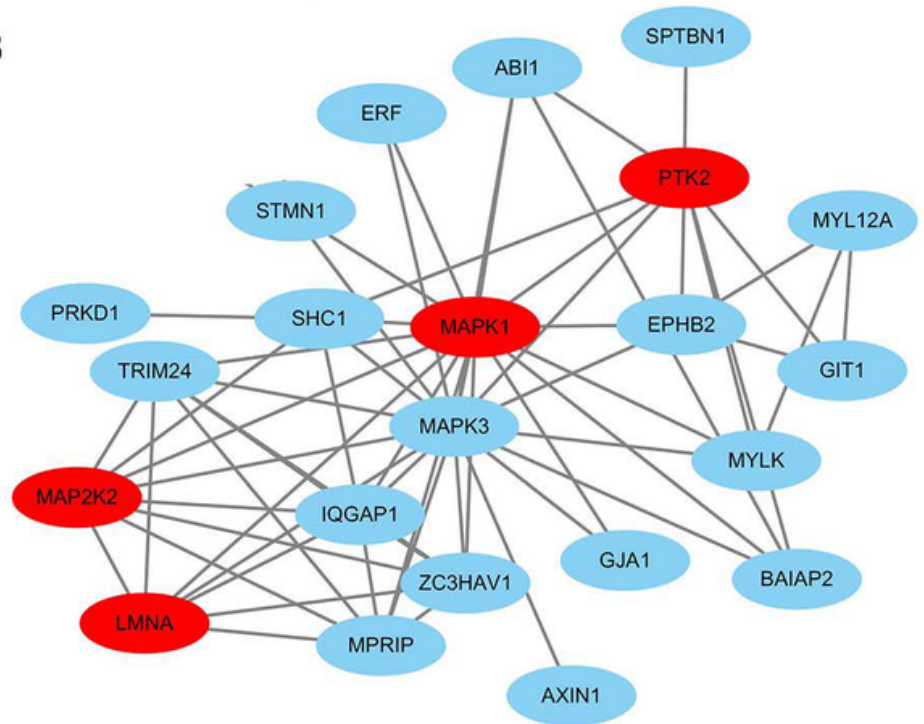

Figure 6

Exhibition of apoptosis-related proteins. 6A:Apoptosis-related proteins centered on HADC1; 6B: Partial apoptosis-related protein interaction network diagram. 


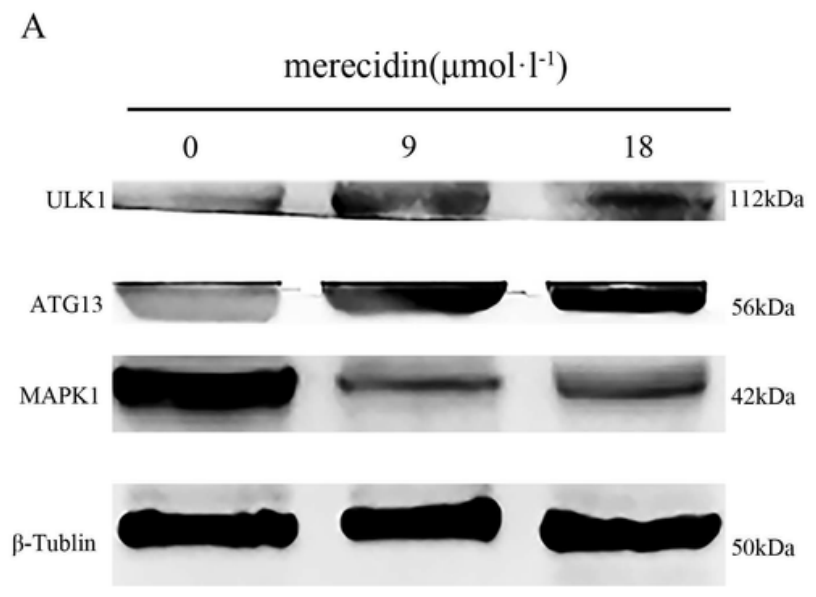

B

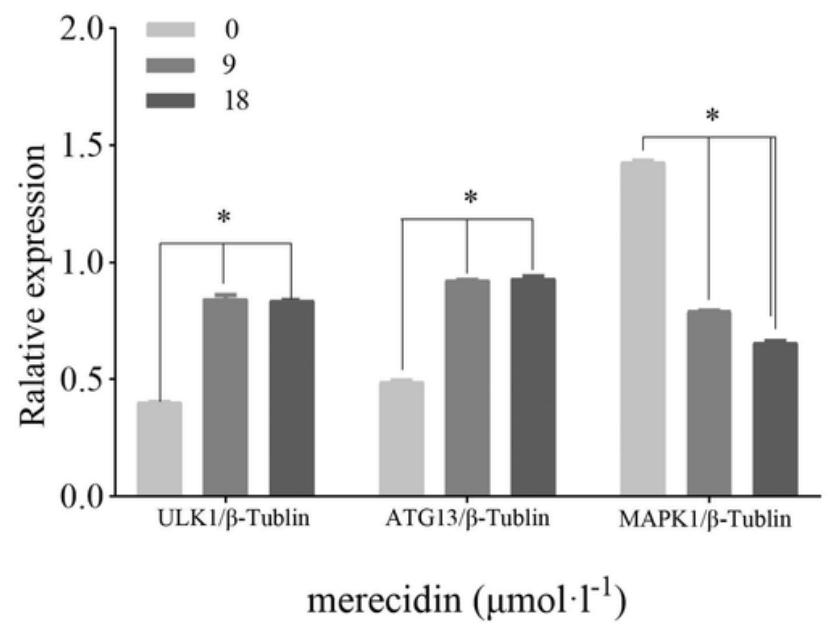

Figure 7

Western blot showed the expression of merecidin-treated A549 cells.

\section{Supplementary Files}

This is a list of supplementary files associated with this preprint. Click to download.

- Supplement.docx

- rawwb.pdf 